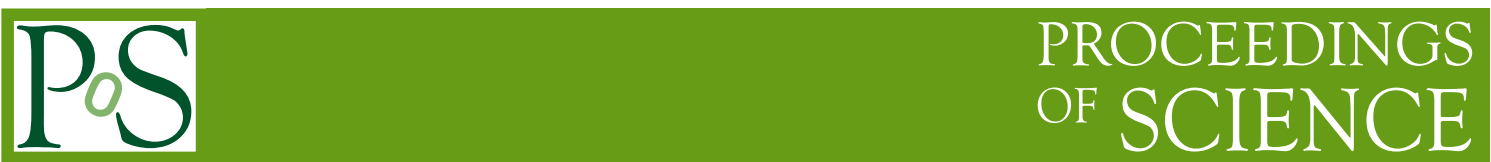

\title{
Measurements of inclusive four-lepton production at ATLAS
}

\author{
Xiaotian Liu*, on behalf of the ATLAS Collaboration \\ University of Science and Technology of China, \\ No.96 Jinzhai Road, Hefei, China \\ E-mail: xiaotian.liu@cern.ch
}

Measurements of differential and integrated fiducial cross-sections in events with two same-flavor, opposite-sign electron or muon pairs are presented with the data corresponding to $139 \mathrm{fb}^{-1}$ of $\sqrt{s}=13 \mathrm{TeV}$ proton-proton collisions collected by the ATLAS dectector during Run 2 of LHC operations (2015-2018). The differential cross-sections exhibit a rich structure with contributions from a number of Standard Model processes. The measurements are performed and compared to state-of-the-art Standard Model calculations, which are found to be consistent with data. The branching ratio of the rare $Z \rightarrow 4 \ell$ decay is extracted. Constraints on a new physics model based on a spontaneously broken B-L gauge symmetry are also evaluated.

40th International Conference on High Energy physics - ICHEP2020

July 28 - August 6, 2020

Prague, Czech Republic (virtual meeting)

${ }^{*}$ Speaker 


\section{Introduction}

Events with final states containing two same-flavor, opposite-sign (SFOS) electron or muon pairs (four leptons) in $p p$ collisions have rich contributions from diverse Standard Model (SM) processes (Figure 1). They are also sensitive to potential beyond-the-SM (BSM) physics. In this proceeding, measurements of four-lepton differential cross-sections and interpretations based on the measured results with ATLAS [1] data collected during Run 2 of LHC (2015-2018) are reported.

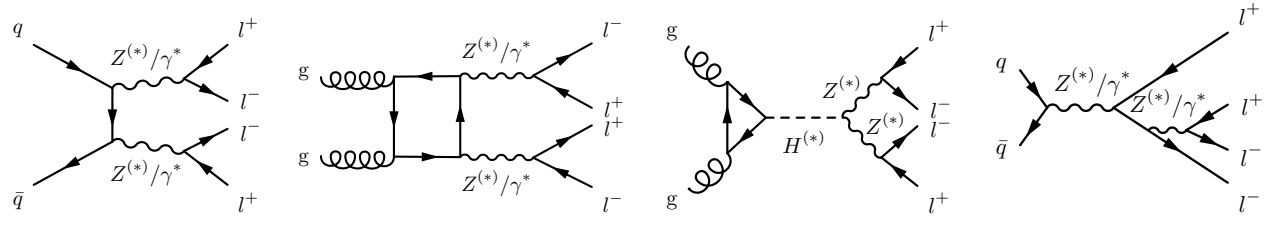

Figure 1: Main SM contributions to the $p p \rightarrow 4 l$ process.

The fiducial phase space is defined according to the acceptance of the ATLAS detector, based on the kinematics of prompt final-state leptons at the particle-level [2,3], and designed to be as inclusive as possible while mitigating background. Electrons are dressed [3] before applying the requirements on transverse momentum $p_{\mathrm{T}}$ and pseudo-rapidity $|\eta|$ in pre-selections [2]. Events are requested to contain at least four such leptons that form two SFOS pairs. In addition, leptons must be isolated with angular separation $\Delta R>0.05$. In order to veto contributions from $J / \psi$ decays, the invariant mass of any SFOS pairs should be larger than $5 \mathrm{GeV}$.

Differential cross-sections are measured as a function of various kinematic variables. Apart from the single-differential measurement of the four-lepton invariant mass, $m_{4 \ell}$, the cross-sections are also measured in slices of the four-lepton transverse momentum, $p_{\mathrm{T}}^{4 \ell}$, the absolute four-lepton rapidity, $\left|y_{4 \ell}\right|$, as well as lepton flavors, i.e. $4 e, 4 \mu, 2 e 2 \mu$ respectively. Other variables are measured in four $m_{4 \ell}$ regions dominated by different processes: single $Z$ boson production $(60<$ $\left.m_{4 \ell}<100 \mathrm{GeV}\right)$, Higgs boson production $\left(120<m_{4 \ell}<130 \mathrm{GeV}\right)$, on-shell $Z Z$ production $\left(180<m_{4 \ell}<2000 \mathrm{GeV}\right)$ and off-shell $\mathrm{ZZ}$ production $\left(20<m_{4 \ell}<60 \mathrm{GeV}\right.$ or $100<m_{4 \ell}<120$ $\mathrm{GeV}$ or $130<m_{4 \ell}<180 \mathrm{GeV}$ ) as well, which are detailed in Ref. [2].

\section{Methods}

The observed data, which are smeared with detector effects including the resolution of the measured variables and the inefficiency of reconstruction, are corrected using a combination of per-lepton efficiency correction and unfolding technique [4]. The correction method is validated to have negligible dependency on the simulation of the underlying kinematics of the particles. The unfolded distributions are compared to particle-level Monte-Carlo (MC) simulation.

The main background comes from events with one or more non-prompt/fake leptons entering the quadruplet and they are mainly from $Z+$ jets and $t \bar{t}$ processes. A data-driven method is used to estimate this background, and it is predicted to be about $4 \%$ of the selected data in the signal region.

The statistical uncertainty on the data is dominant for most measured bins. For the systematic uncertainties, dominant contributions arise from uncertainties on lepton efficiency, resolution and energy or momentum scale. The other sources of uncertainty including luminosity, theoretical modelling, as well as background are all considered [2]. 


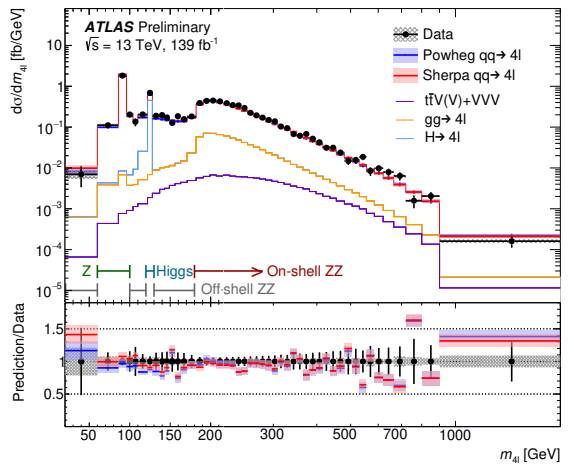

Figure 2: Differential cross-section of $m_{4 \ell}$. The breakdown of contributions from different SM processes is also shown [2].

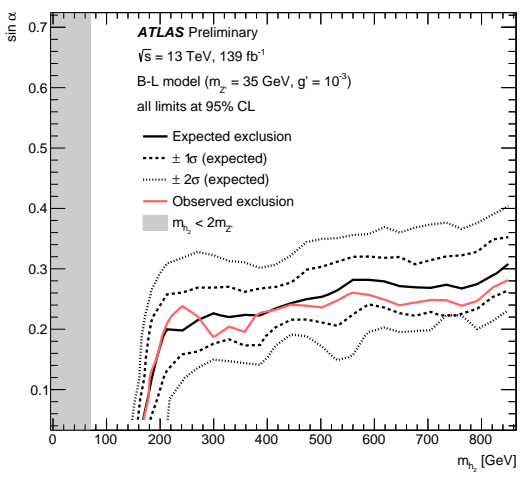

Figure 3: Exclusion contour at $95 \%$ confidence level for the B-L model in the plane of $\sin \alpha$ versus $m_{\mathrm{h}_{2}}$ for $m_{\mathrm{Z}^{\prime}}=35 \mathrm{GeV}$ and $g^{\prime}=10^{-3}$ [2].

\section{Results}

The differential cross-section as a function of $m_{4 \ell}$ is shown in Figure 2. The SM predictions agree well with the measurement within uncertainties over the entire $m_{4 \ell}$ spectrum. Differential measurements for other variables can be found in Ref. [2].

The branching ratio of the rare process $Z \rightarrow 4 \ell$ is extracted with the measured cross-section in the single $\mathrm{Z}$ region: $\mathcal{B}_{\mathrm{Z} \rightarrow 4 \ell}=(4.41 \pm 0.30) \times 10^{-6}$ [2].

Constraints on a model based on a spontaneously broken B-L gauge symmetry [5] are also evaluated. The 95\% confidence exclusion contour is shown in Figure 3, in the plane of $\sin \alpha$ and $m_{\mathrm{h}_{2}}$.

\section{References}

[1] ATLAS Collaboration, The ATLAS Experiment at the CERN Large Hadron Collider, JINST 3 (2008) S08003.

[2] ATLAS Collaboration, Measurements of differential cross-sections in four-lepton events in $13 \mathrm{TeV}$ proton-proton collisions with the ATLAS detector, ATLAS-CONF-2020-042, URL: https://cds.cern.ch/record/2728051.

[3] ATLAS Collaboration, Proposal for truth particle observable definitions in physics measurements, ATL-PHYS-PUB-2015-013, URL: https://cds.cern.ch/record/2022743.

[4] G. D'Agostini, A multidimensional unfolding method based on Bayes' theorem, Nucl. Instrum. Meth. A 362 (1995) 487.

[5] F.F. Deppisch, W. Liu and M. Mitra, Long-lived heavy neutrinos from Higgs decays, JHEP 08 (2018) 181. 